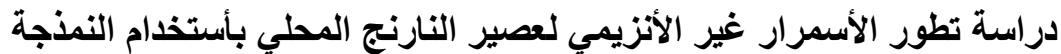

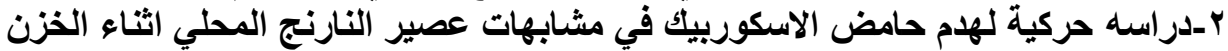

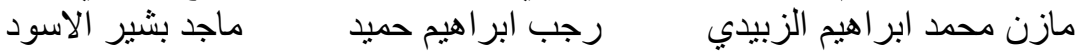

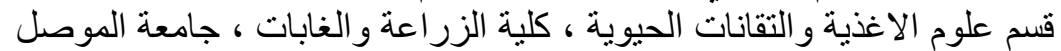

\section{الخلاصة}

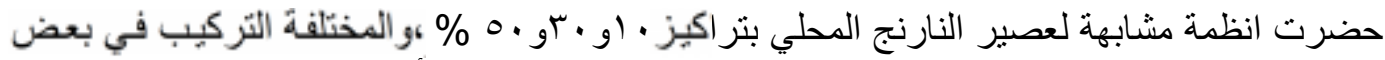

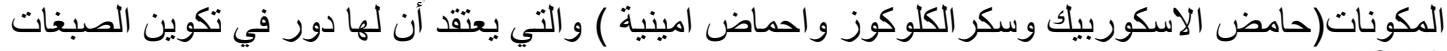

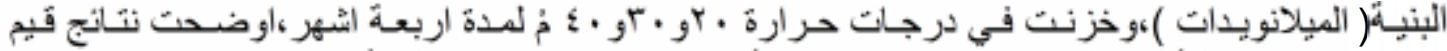

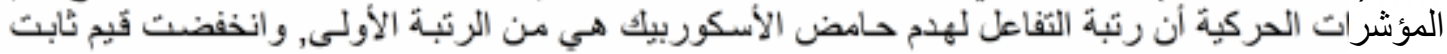

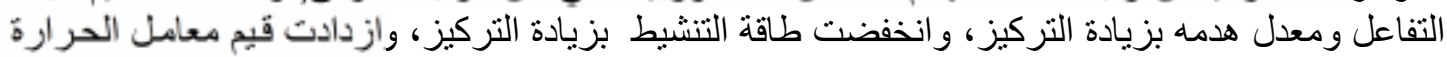

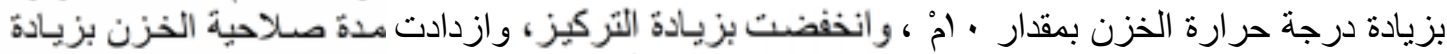

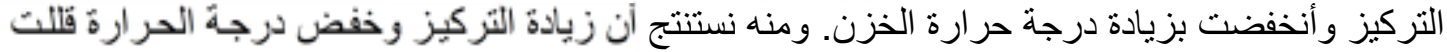

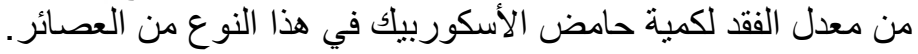

\section{المقدمة}

تعد ثمار الحمضيات من الفواكه المنتشرة زر اعتها عالميا ، اذ تمثل مركز ا مهما في التجارة الدولية (اغـا

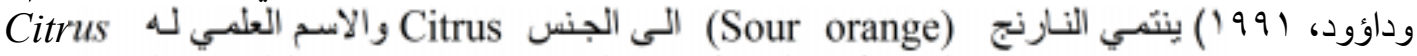

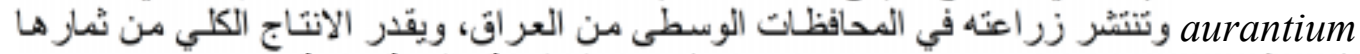

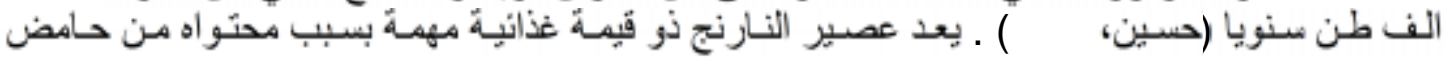

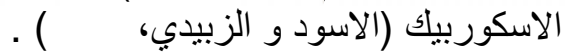

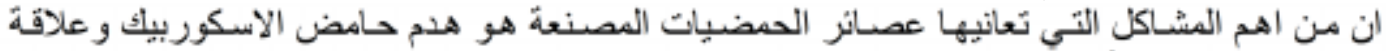

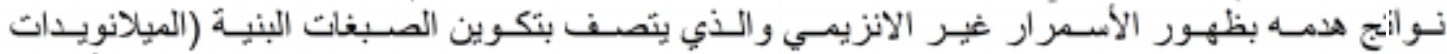

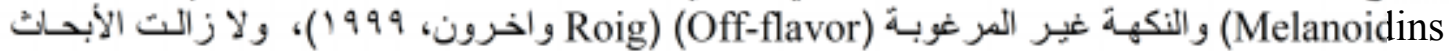

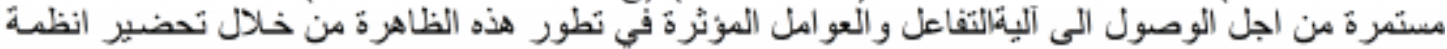

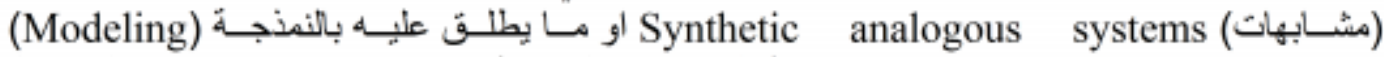

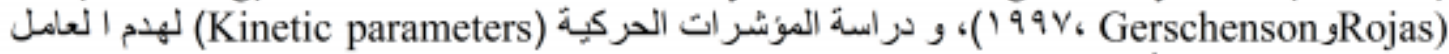

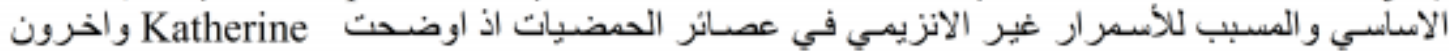

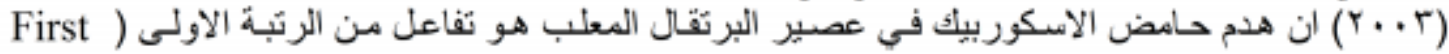

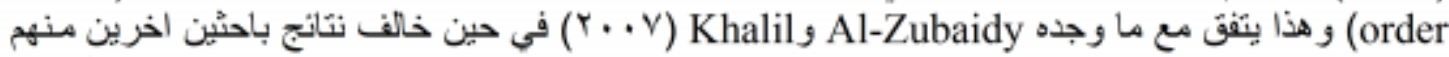

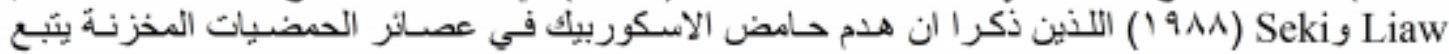

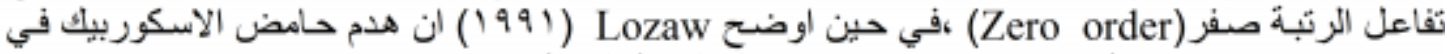

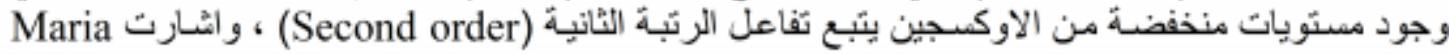

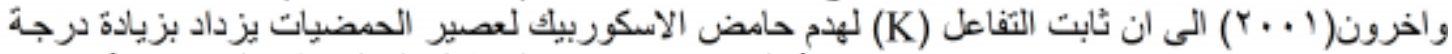

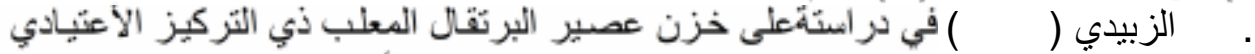

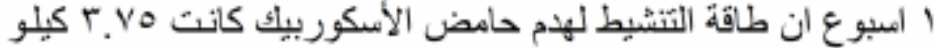

Khalil Al-Zubaidy

ت ی

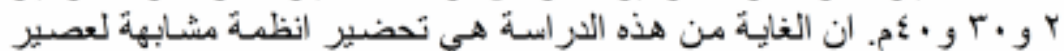

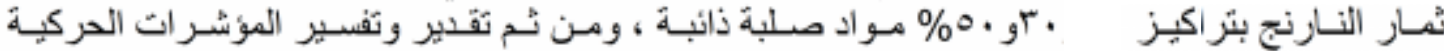

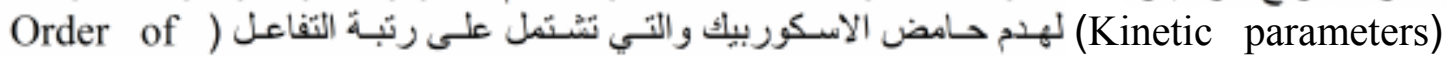

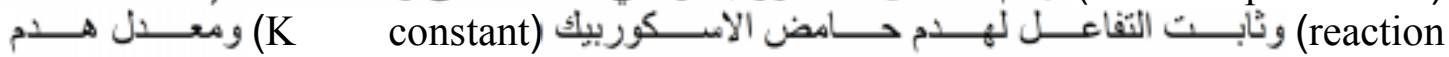

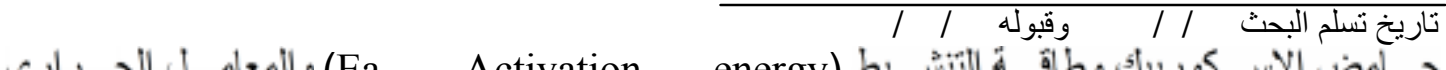

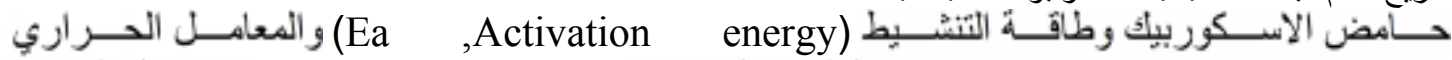

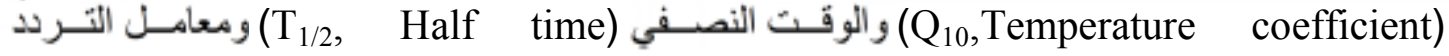



( ) ( )
(ISSN 1815-316X)
ر رافين

لهذه الانظمـة بالاعتمـاد على (Shelf- life) و إيجـاد مـد صـلاحية الخزن (A,Frequency factor) محتو اها من حامض الأسكوربيك.

\section{مواد البحث وطر ائقه}

عصير تُــار النـارنج المحلي

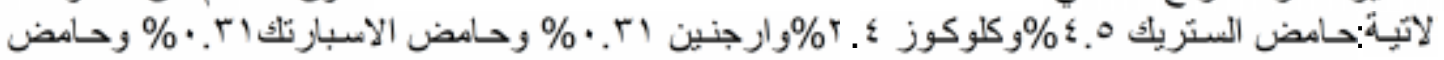

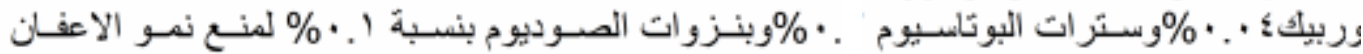
كيز ( ) C Christine Gordon)

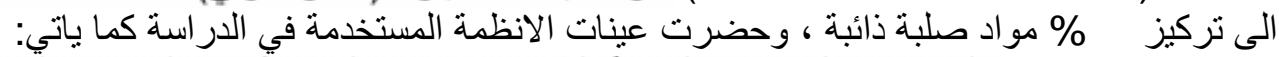

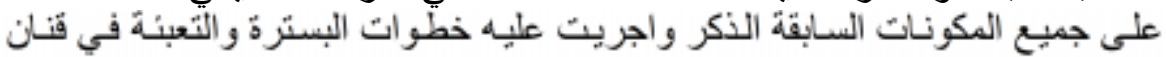

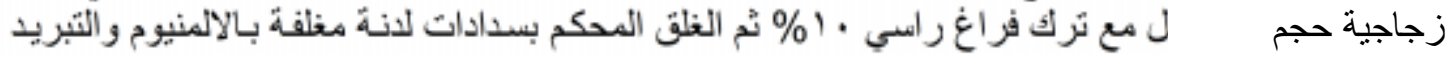

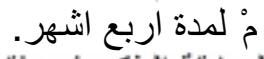

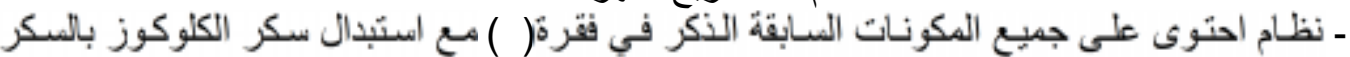

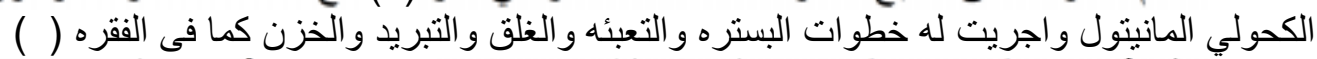

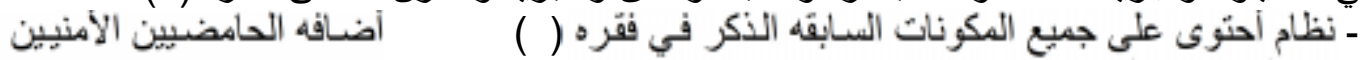

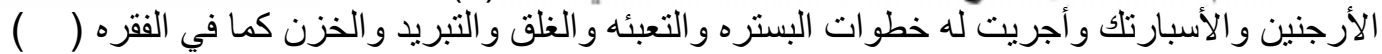

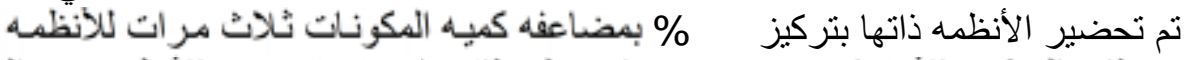

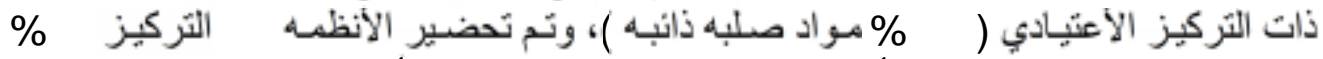

$$
\text { اعفه تركي }
$$

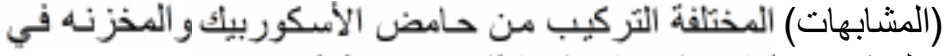

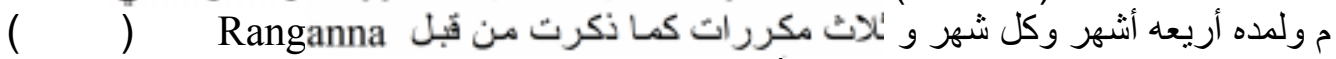

تم تقدير قيم المؤشر ات الحركيه لهدم حامض الأسكوربيك

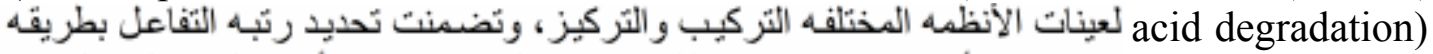
Nuray ،
) Excel

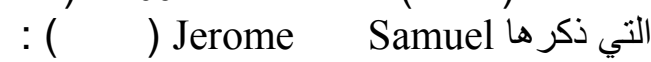
لهدم حامض الأسكوربيك وطبقا لما ذكره Nuray أخرن $-\mathrm{Ea}$

Slope $=\frac{}{2.303 * \mathrm{R}}$

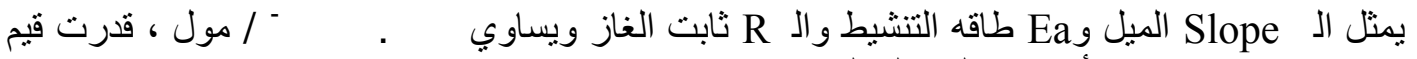
$\mathrm{Q}_{10}=\left(\mathrm{K}_{2} / \mathrm{K}_{1}\right)^{10 / \mathrm{T}_{2}-\mathrm{T}_{1}}$

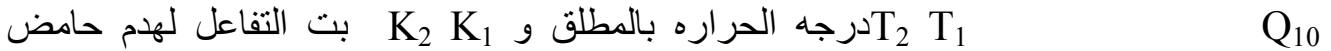
تم أيجادوبيك

$\mathrm{T}_{1 / 2}=\ln (2) / \mathrm{K}$

$=0.693 / \mathrm{K}$

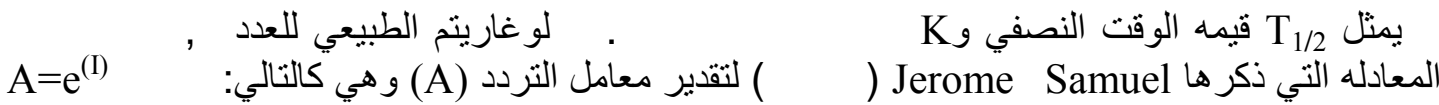

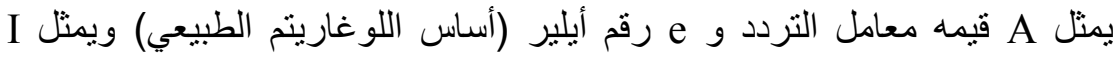

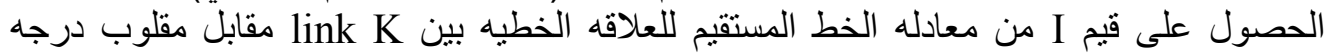
- Excel 
( )Khalil Al- Zubaidy Visual Basic

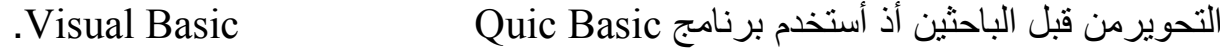

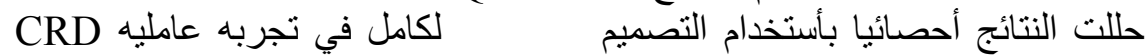

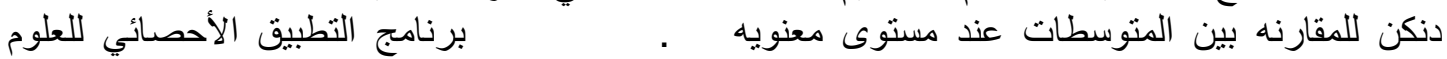

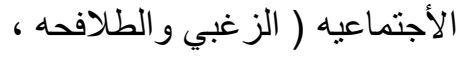

\section{النتائج والمناقشة}

1ـ المؤشرات الحركية لهدم حامض الاسكوربيك:

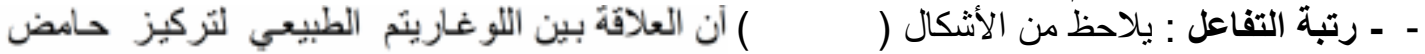

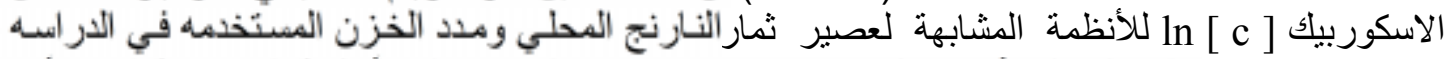

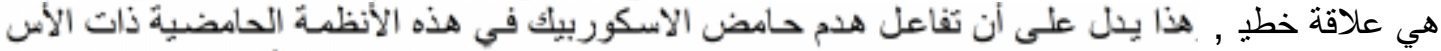

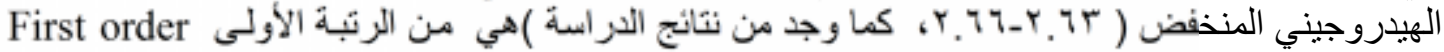
هohnson) (reaction)

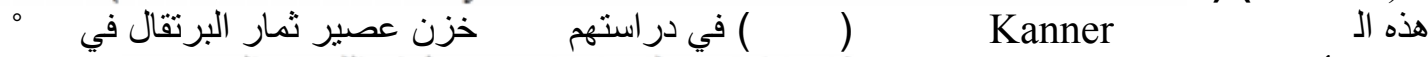

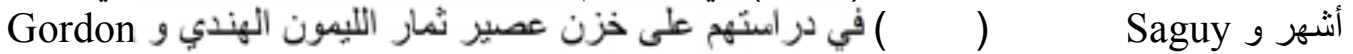
Christine

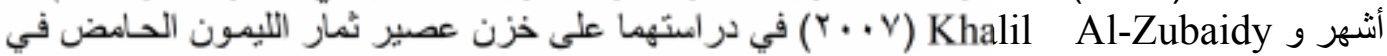

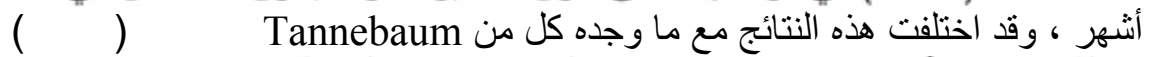
Sebi Liao

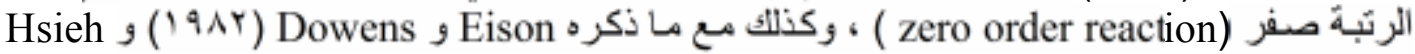

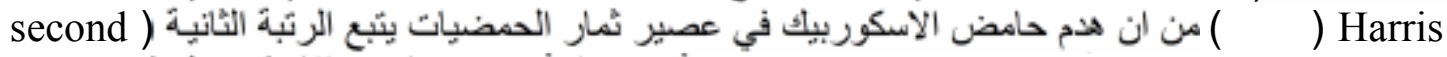

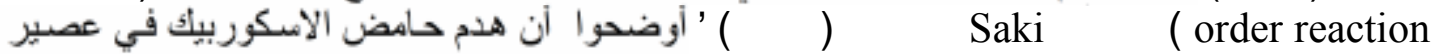

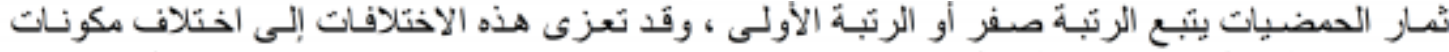

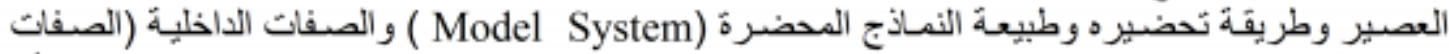

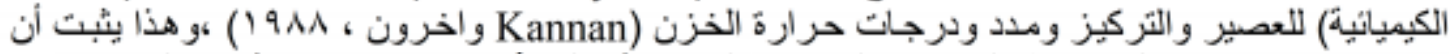

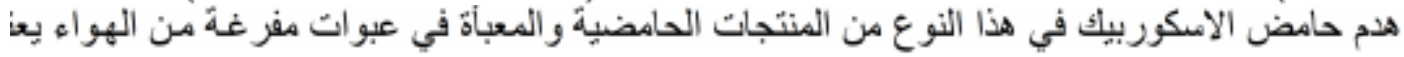

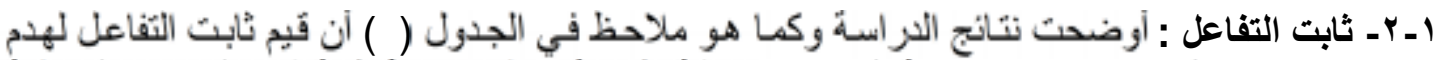

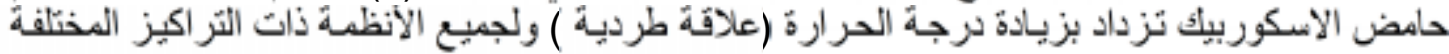
المستخدمة في الدر اسة ، إذ بلغت القيم للنظام المتكامل ذبي التركيز

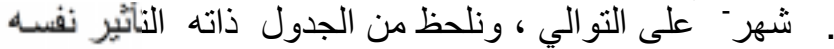

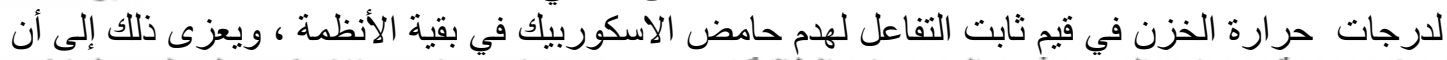

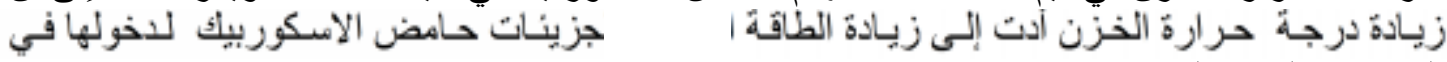

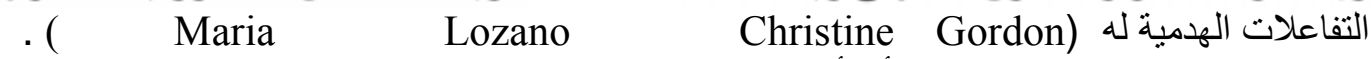
بينت النتائج من الجدول ذاته أن أعلى القيم لثابت التفاعل لهدم حامض الاسكوربيك كانت في النظام

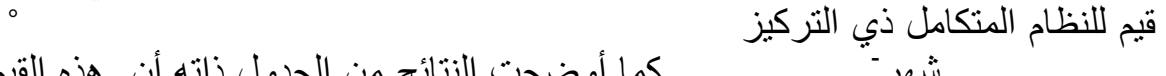

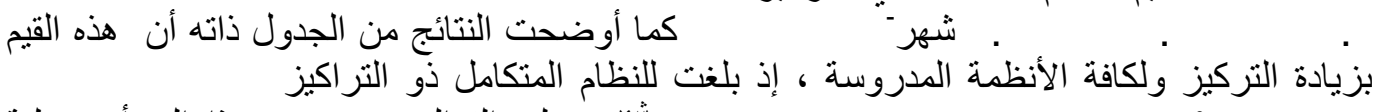

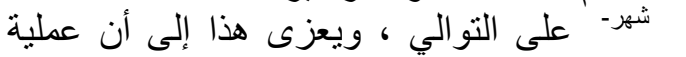

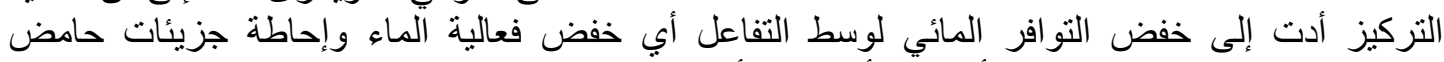

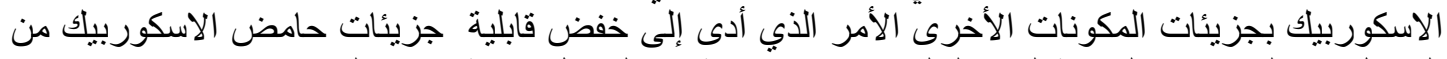

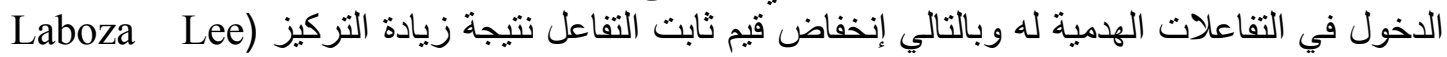

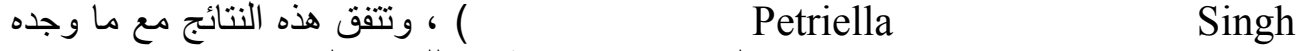

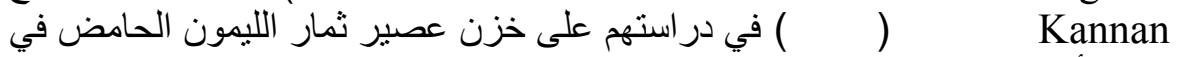

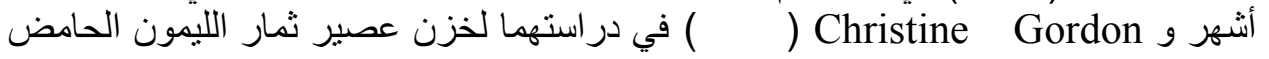



( ) ( )
ر رافين

Gerschenson Rojas

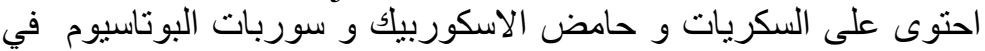

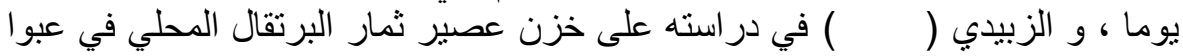
زجاجية ومعدنية في
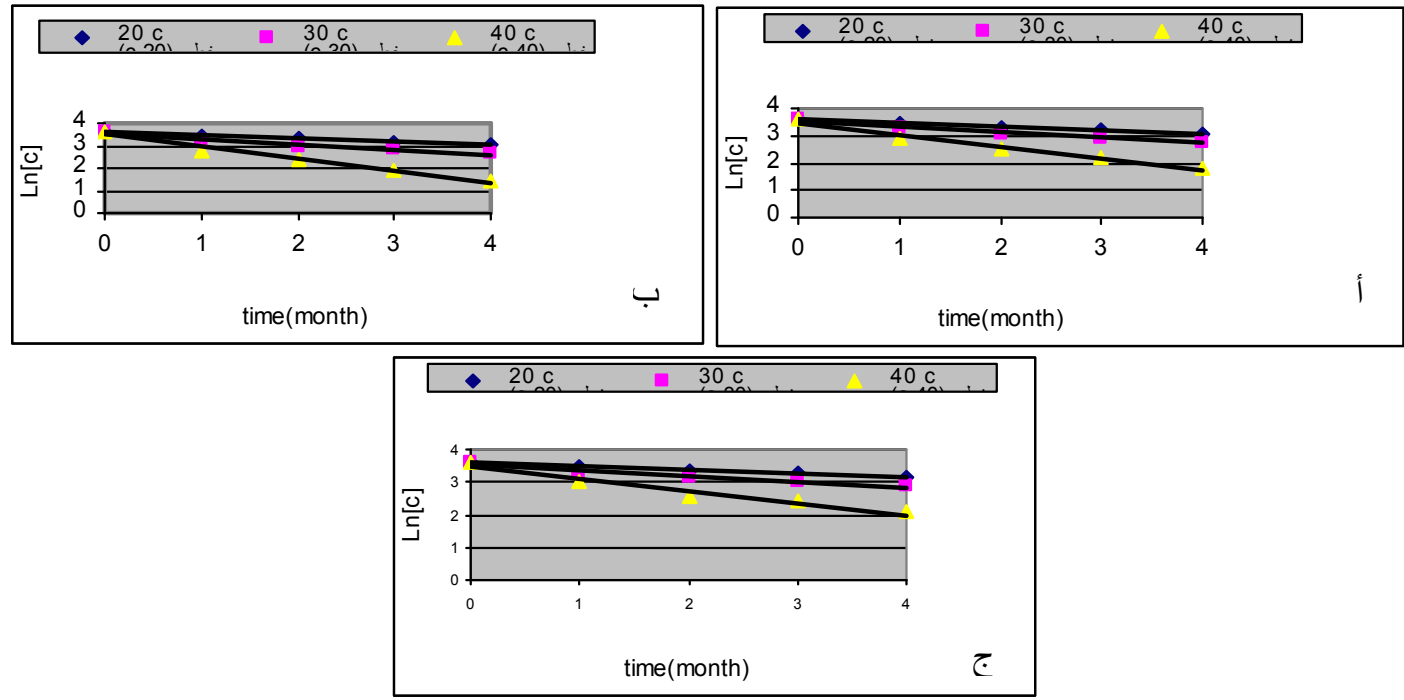

( ) : العلاقة بين اللوغاريتم الطبيعي لتركيز حامض الأسكوربيك ومدة الخزن للنظام الكامل تركيز
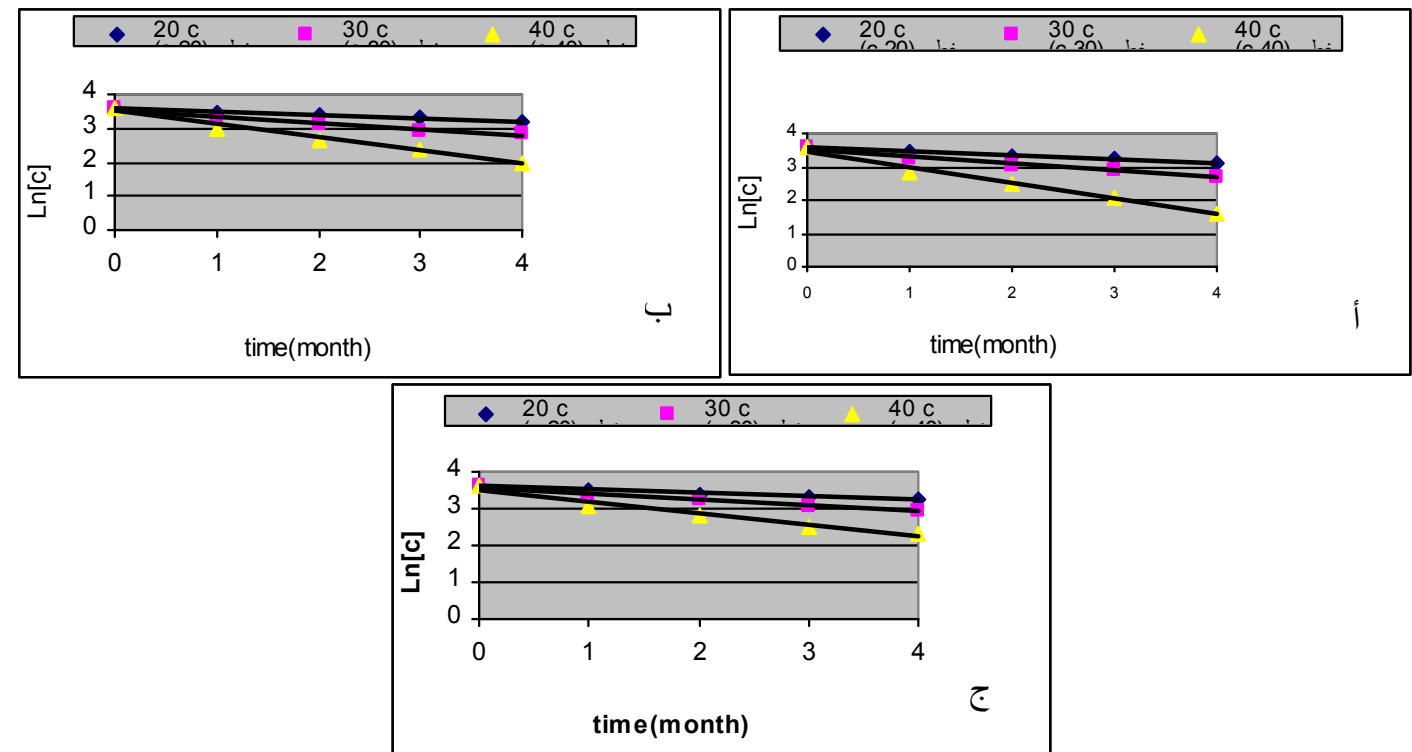

( ) ) العلاقة بين اللو غاريتم الطبيعي لتركيز حامض الأسكوربيك ومدة الخزن للنظام بدون

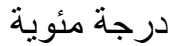
( )\% ( )\%

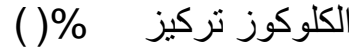



( ) ( )
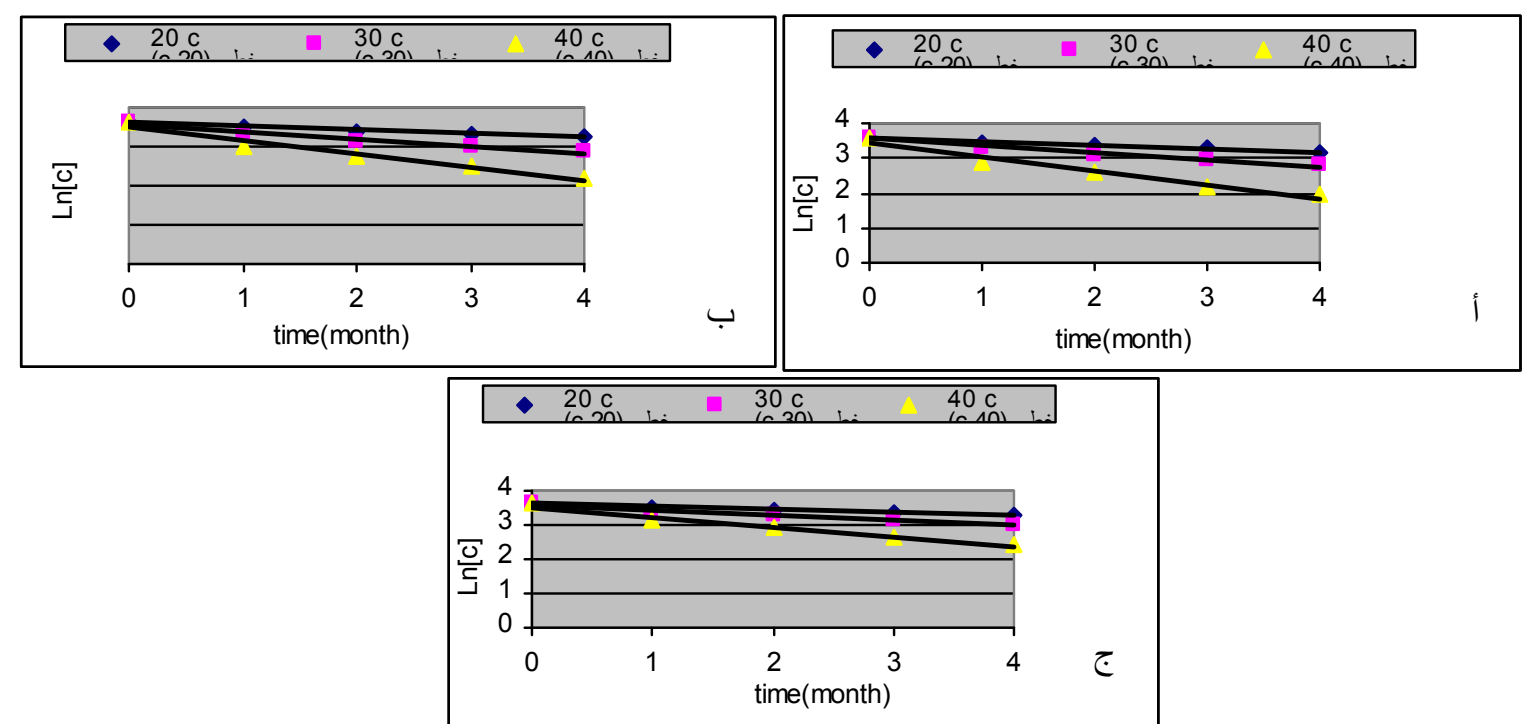

( ) ) العلاقة بين اللوغاريتم الطبيعي لتركيز حامض الأسكوربيك ومدة الخزن للنظام بدون
درجة مئوية
( )\%
( )\%

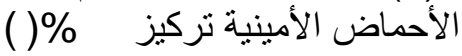

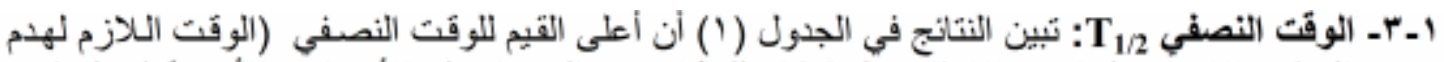

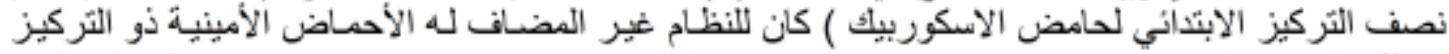

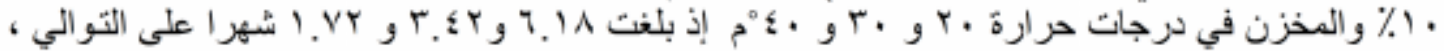

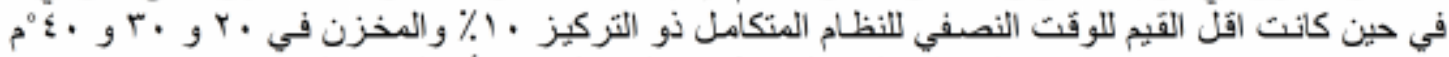

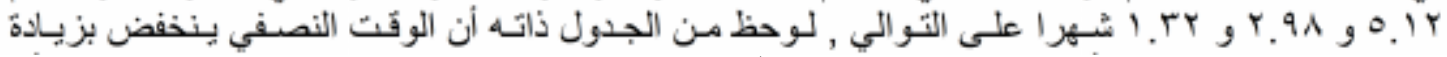

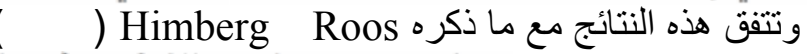

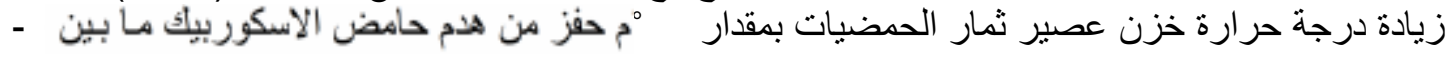

تبين النتائج من الجدول ذاته أن الوقت النصفي داد بزيادة التركيز وللأنظمة كافة إذ بلغت قيمه للنظام

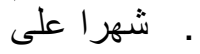

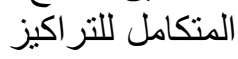

التو الي ، وهذا يؤكد ما ذكره Navarro

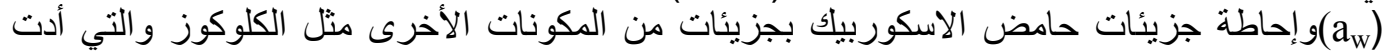

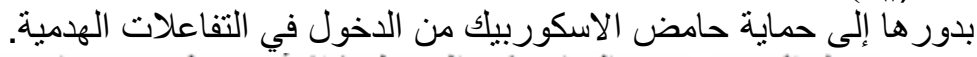

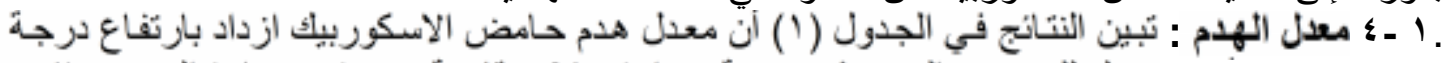

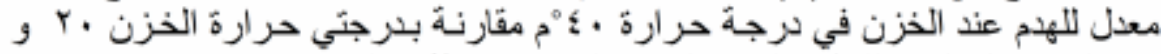

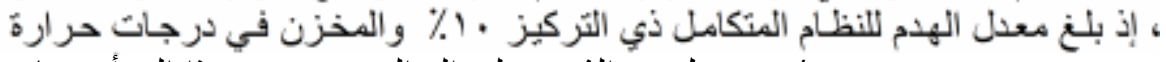

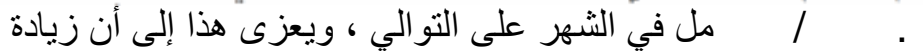

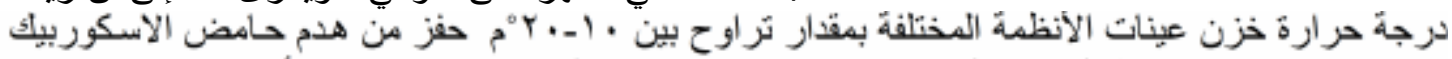

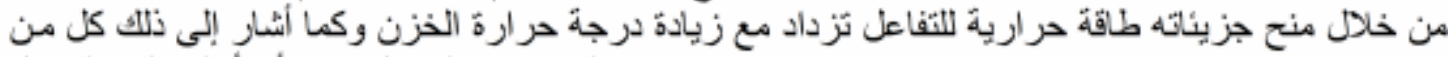

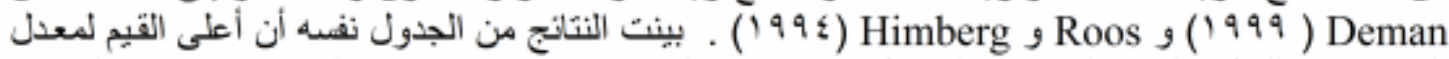

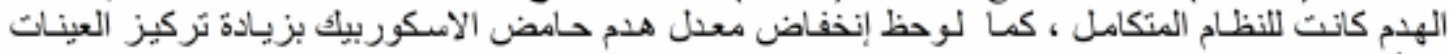

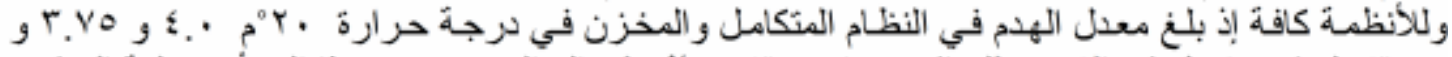

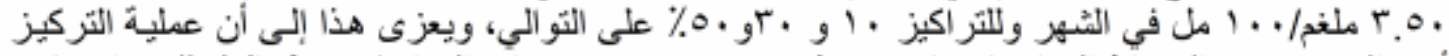

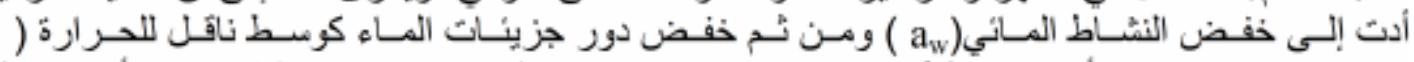

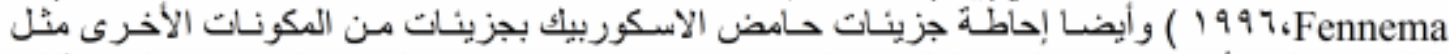

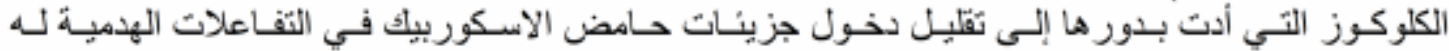

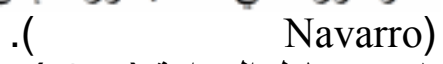

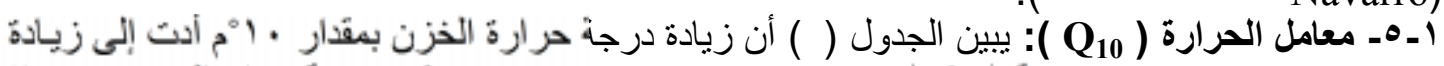

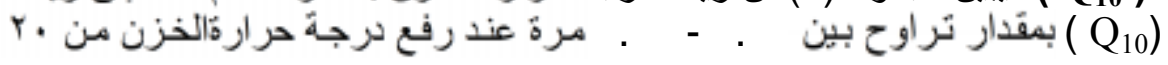



( ) ( )
(ISSN 1815-316X)
رافدين

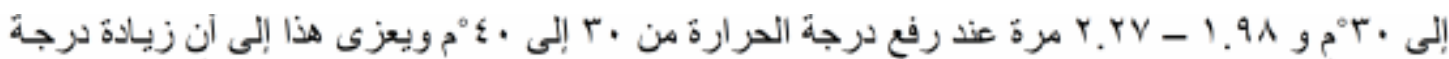

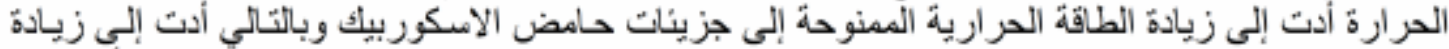

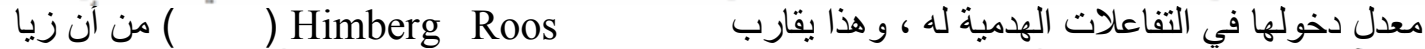

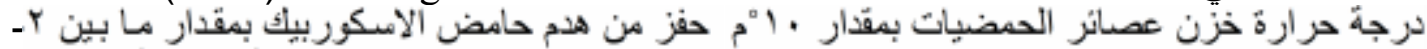

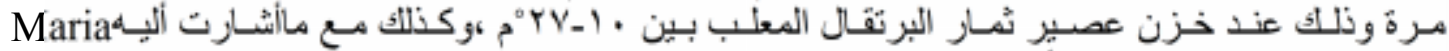

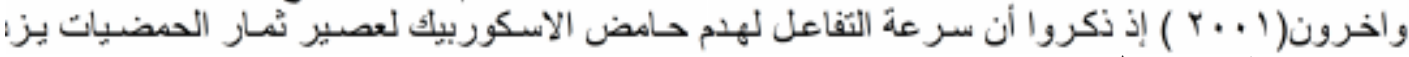

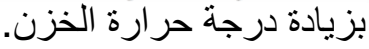

( ) :ثابت التفاعل والوقت النصفي ومعدل الهدم ومعامل الحرارة لهدم حامض الاسكوربيك للانظمة

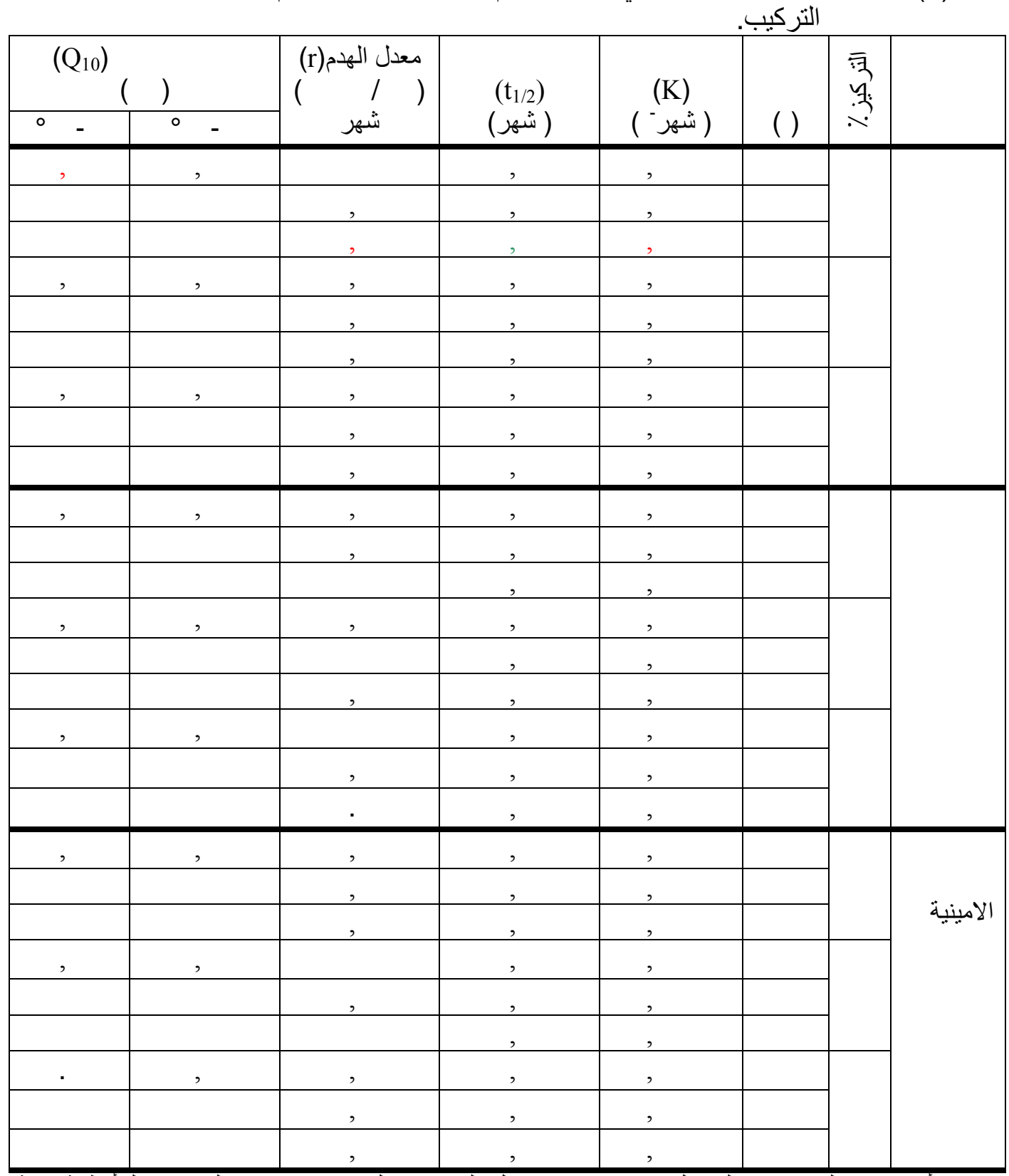

أوضحت النتائج في الجدول ذاته إن قيم معامل الحرارة تقل بزيادة تركيز العينات وللانظمة كافة ،

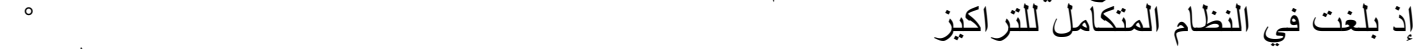

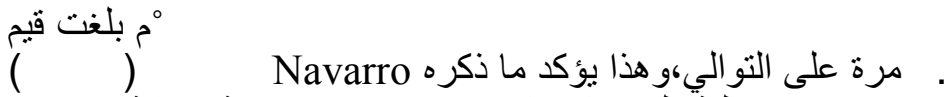

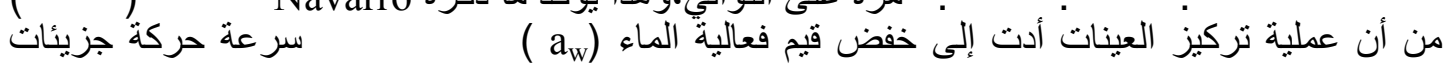



( ) ( )
(ISSN 1815-316X)
رافدين

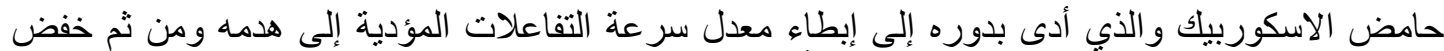

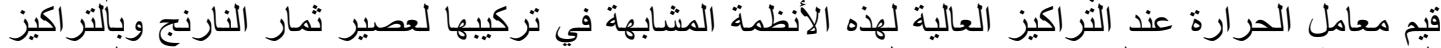

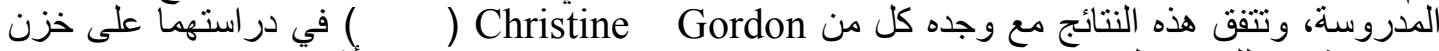

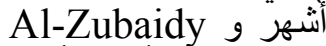
مْم لمدة أربعة أثنهر.

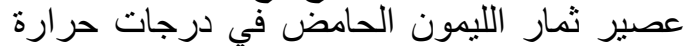
Khali

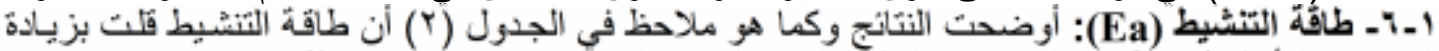

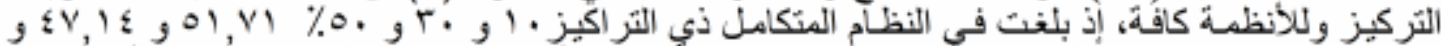
المبكو, YV

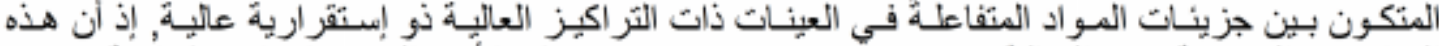

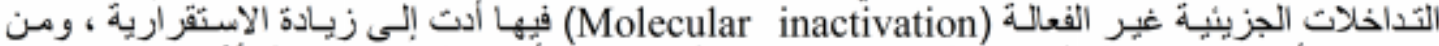

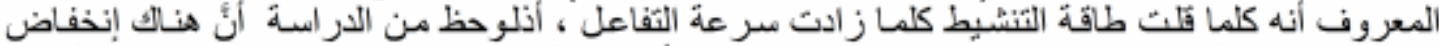

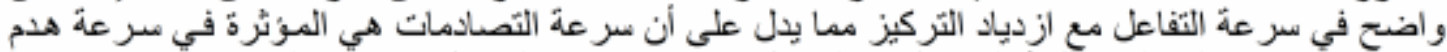

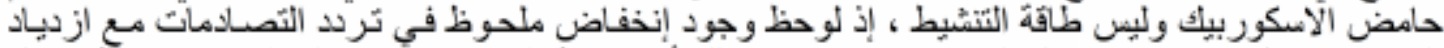

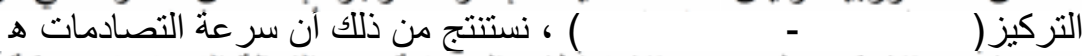

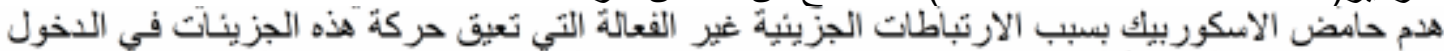

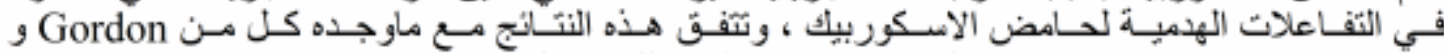
Christine

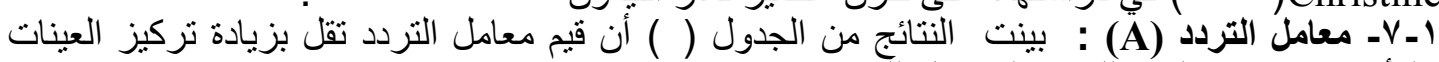

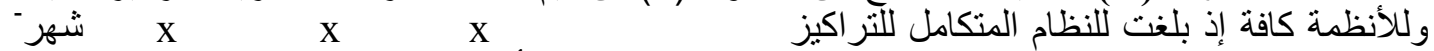

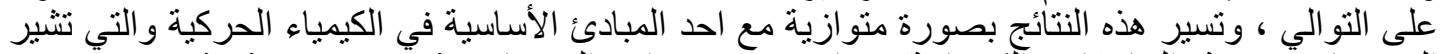

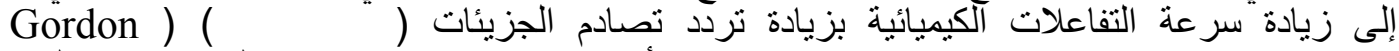
Christine

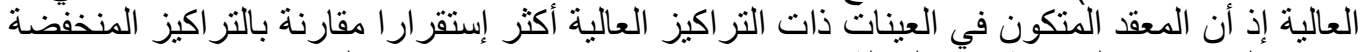

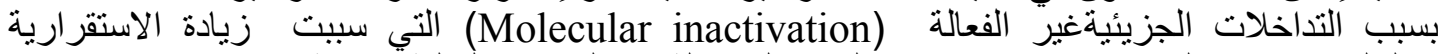
وبالتالي إنخفاض التصادمات بين جزيئات المواد المتفاعلة في التر اكيز العالية ومن ثم إنخفاض هدم الاسكوربيك .

( ): نتائج تطبيق معادلة ارهينيوس على البيانات الحركية لهام حامض الاسكوربيك للانظمة

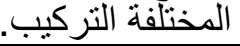

\begin{tabular}{|c|c|c|c|c|c|c|}
\hline 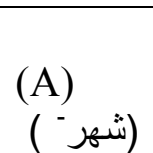 & 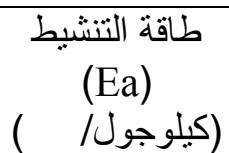 & (r) & & الميل & التركيز & \\
\hline$x$ & . & , & , & . - & & \\
\hline $\mathrm{x}$ & . & , & , & . - & & \\
\hline $\mathrm{X}$ & . & , & . & . - & & \\
\hline $\mathrm{X}$ & . & . & . & $. \quad-$ & & \\
\hline $\mathrm{X}$ & . & . & . &. & & \\
\hline $\mathrm{X}$ & . & . & . & . - & & \\
\hline $\mathrm{X}$ & . & . & . & . - & & \\
\hline $\mathrm{x}$ & . & . & . & $. \quad-$ & & \\
\hline $\mathrm{X}$ & . & . & . & . - & & مينية \\
\hline
\end{tabular}

بينت النتائج من الجدول ذاته أن اقل القيم لمعامل التردد كانت في النظام غير المضاف له الأحماض

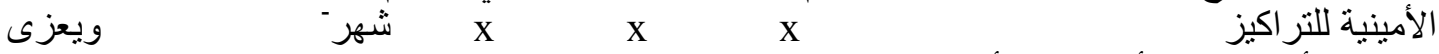
ذللك إلى أن تفاعل الأحماض الأمينية مع المجاميع المختزلة لنواتج الهُم الفئل الفعالة غير معنوي في البيئات

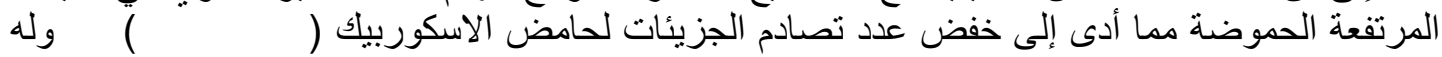
في التفاعلات الهدمية .

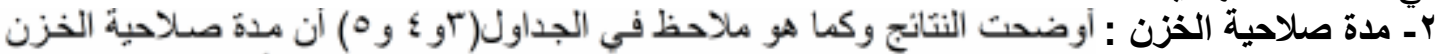

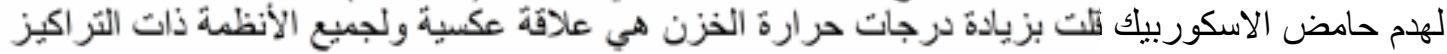
المتكامل ذي التركيز 

( ) ( )
(ISSN 1815-316X)
ر رافين

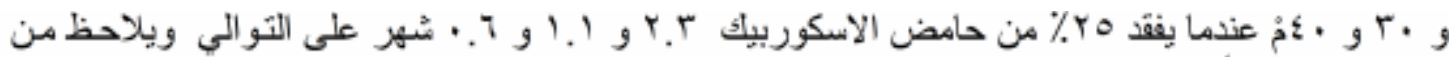

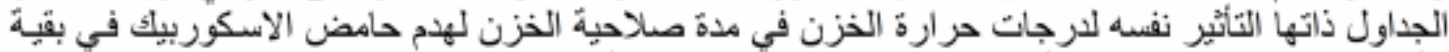

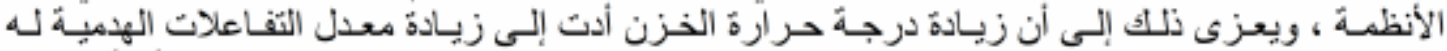

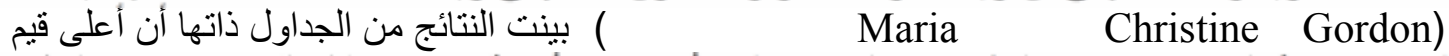

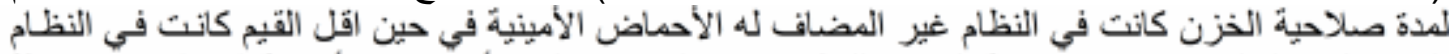

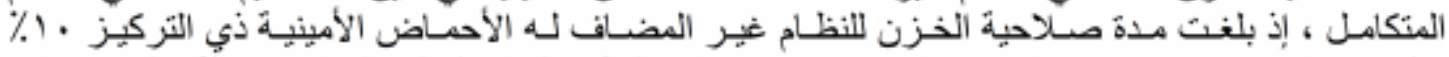

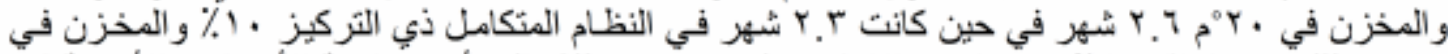

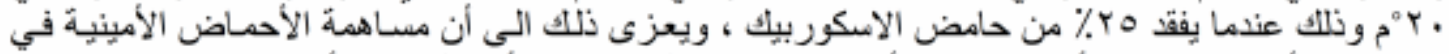

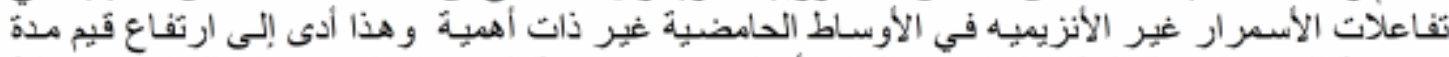

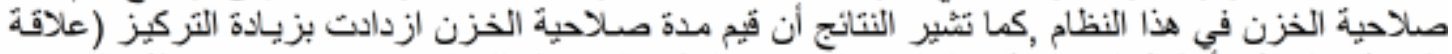

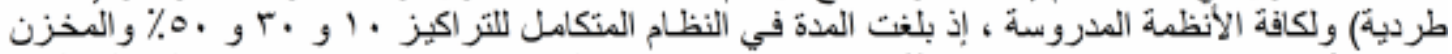

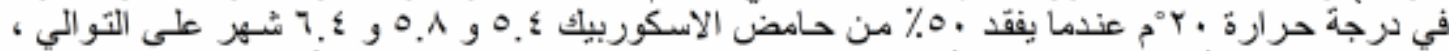

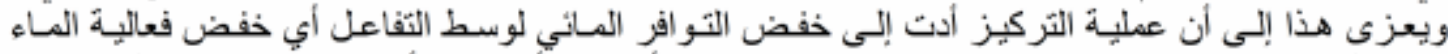

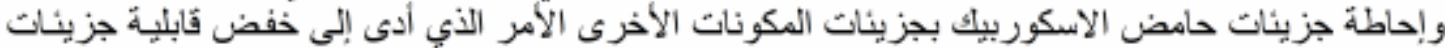

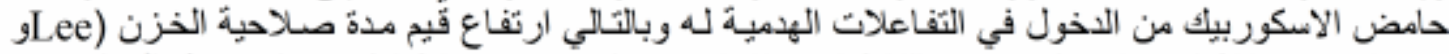
Laboza

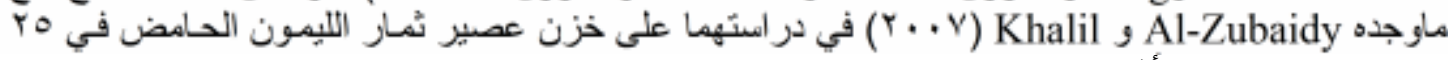
أَشهر.

( ):مدة صلاحية الخزن للنظام الكامل بالعلاقة مع محتو اه من حامض الاسكوربيك.

\begin{tabular}{|c|c|c|c|c|c|c|}
\hline \multicolumn{6}{|c|}{ التركيز } & \multirow[b]{2}{*}{ ( ) } \\
\hline \multicolumn{6}{|c|}{ الفقد في حامض الاسكوربيك (شهر ) } & \\
\hline . & . & . & . & . & . & - \\
\hline . & . & . & . & . & . & - \\
\hline . & . & . & $\dot{.}$ & . & . & - \\
\hline . & . & . & . & . & . & \\
\hline . & - & . & . & . &. & \\
\hline . & . & . & . & . &. & \\
\hline . & . & . & . & . & . & \\
\hline - & . & . & . & . & . & \\
\hline . & . & . & . & . & . & \\
\hline$\cdot$ & . &. & . &. &. & \\
\hline$\cdot$ & . & . & . & . &. & \\
\hline$\dot{ }$ & . & . & . & . & . & \\
\hline 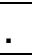 & $\therefore$ & . & . &. &. & \\
\hline . & - & 8 & . & 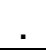 & . & \\
\hline
\end{tabular}



()$\quad(\quad)$
(ISSN 1815-316X)
ر رافين

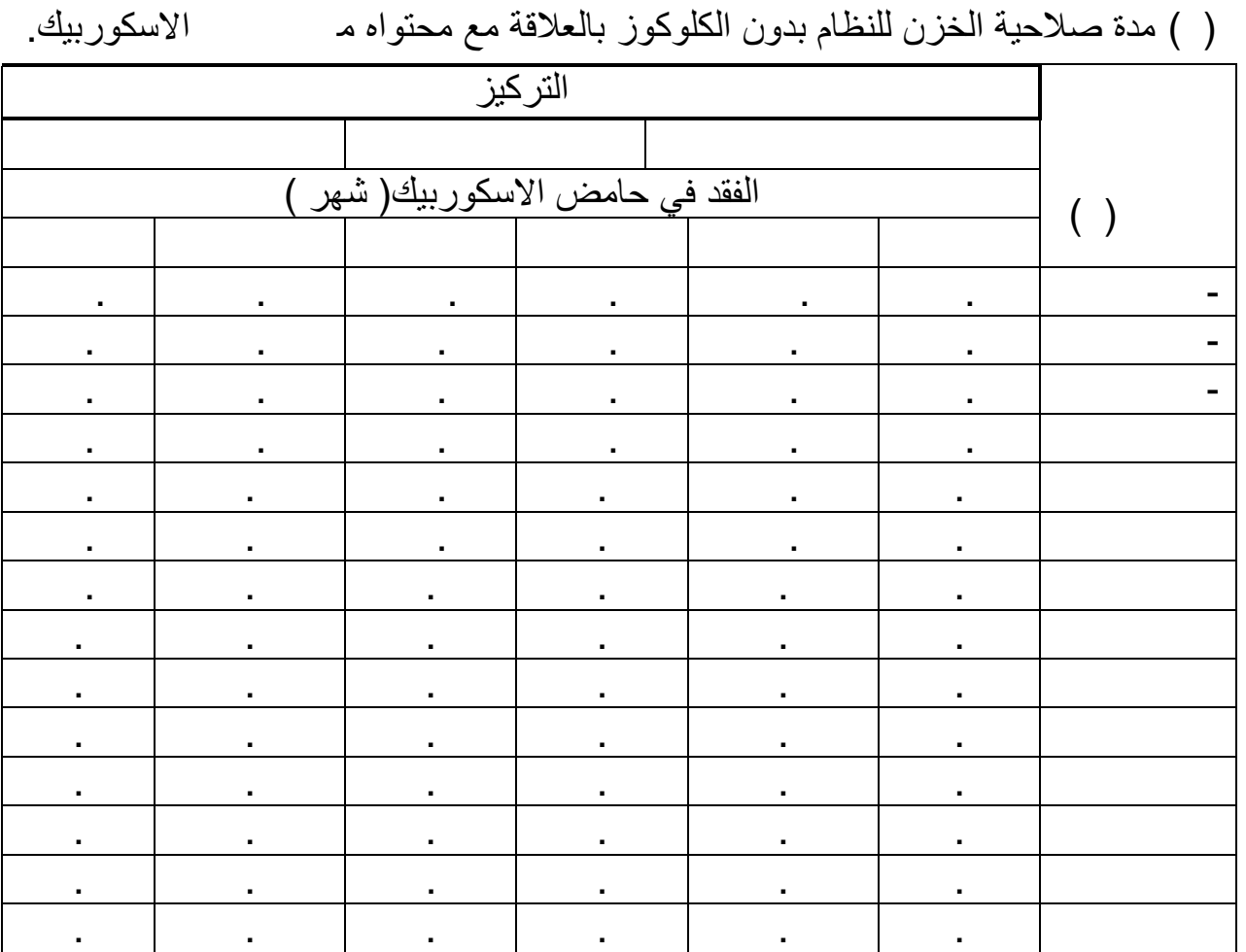

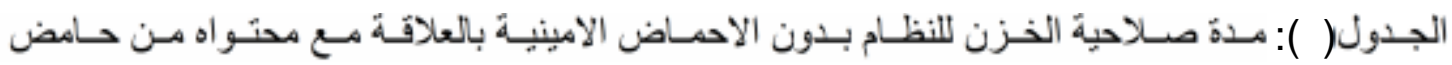
الاسكوربيك

\begin{tabular}{|c|c|c|c|c|c|c|}
\hline \multicolumn{6}{|c|}{ التركيز } & \multirow{3}{*}{ ( ) } \\
\hline \multicolumn{6}{|c|}{ الفقد في حامض الاسكوربيك(شهر ) } & \\
\hline & & & & & & \\
\hline . & . & . & . & . & . & - \\
\hline . & . & . & . & . & . & - \\
\hline . & . & . & . & . & . & - \\
\hline . & . & . & . & . & . & \\
\hline . & - & . & . & . & . & \\
\hline . & . & . & . & . & . & \\
\hline . & . & . & . & . & . & \\
\hline . & . & · & . & . & . & \\
\hline . & . & . & . & . & . & \\
\hline$\cdot$ & . & . & . & . & . & \\
\hline . & . & . & . & . & . & \\
\hline$\dot{.}$ & . & . & . & . & . & \\
\hline . & $\cdot$ & . & . & . & . & \\
\hline . & . & . & . & . & ${ }^{2}$ & \\
\hline
\end{tabular}



( ) ( )
(ISSN 1815-316X)
رافين

\title{
STUDY OF NONENZYMATIC BROWNING DEVELOPMENT OF LOCAL SOUR ORANGE JUICE BY USING MODEL SYSTEMS \\ 2-KINETIC STUDY OF ASCORBIC ACID DEGRADATION IN MODEL SYSTEMS OF LOCAL SOUR ORANGE JUICE DURING STORAGE.
}

Mazin M. Al-Zubaidy Rajab I. Al-Doskey Majid B. Al-Aswad

Food Sci. and Biotech. Dept. College of Agric. \& Forestry, Mosul Univ., Iraq

\begin{abstract}
Synthetic model systems of local sour orange juice prepared of concentrations 10,30 and 50\%, with different contents of some compounds (ascorbic acid, glucose and amino acids) that might play a role in brown pigments formation. Samples were stored at 20,30 and 40 C for 4 months. Results of kinetic parameters of ascorbic acid degradation showed that the reaction order was first order, and the value of reaction constant $(\mathrm{k})$ and degradation rate increased with increasing concentration of model system. The highest value of activation energy for ascorbic degradation was found in model system contained all compounds mentioned above and decreased with increasing concentration. Temperature coefficient $\left(\mathrm{Q}_{10}\right)$ increased with increasing storage temperature, The shelf life of model systems increased with increasing concentration and decreased with increasing storage temperature.

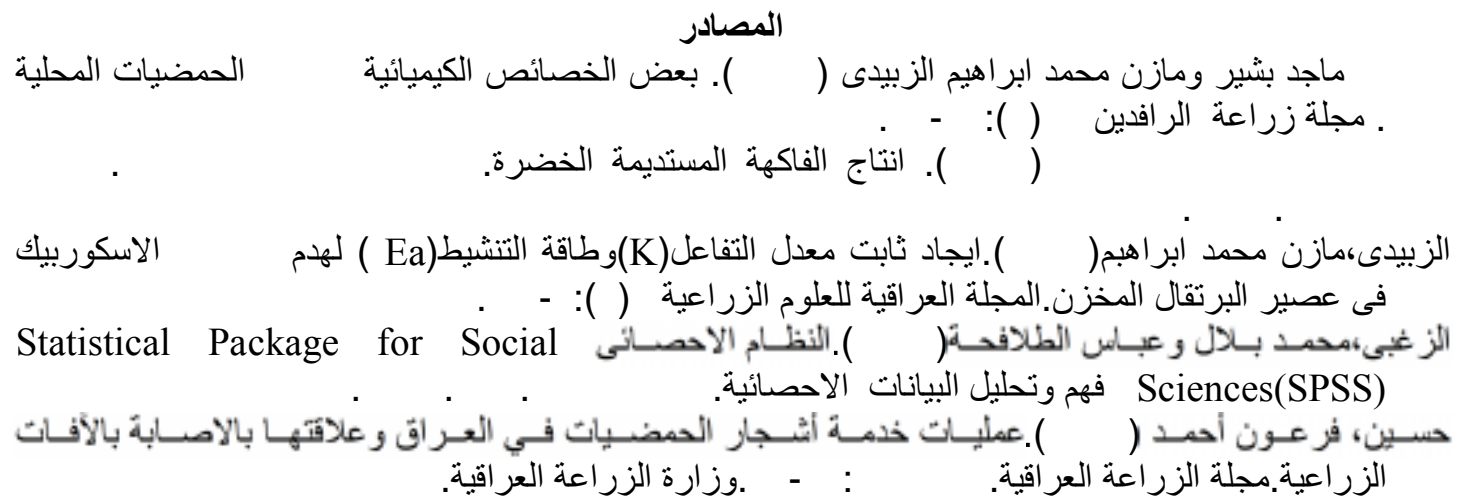

Al-Zubaidy,M.and A.Khalil (2007).Kinetic and predicition studies of ascorbic acid degradation in normal and concentrate local lemon juice during storage. Food Chemistry.101:254-259.

Deman, J. (1999). Principles of Food Chemistry ( $2^{\text {nd }}$ ed.). AV1 Pub. Co., Westport, CT.

Eison-Perchonok, M. and T. Dowens (1982). Kinetic of ascorbic acid autoxi-dation as a function of dissolved oxygen concentration and temperature. J. Food Sci. 47:765767.

Fennema, O. (1999). Food Chemistry. Marcel Dekker. Inc., New York.

Gordon, L. and S. Christine (1990). Effect of soluble solids and temperature on ascorbic acid degradation in lemon juice storaged in glass bottles. J. Food Quality.13:361373.

Houston, P. (2001). Chemical Kinetics and Reaction Dynamics. Singapore: McGraw Hill.

Hsieh, Y. and N. Harris ( 1993 ). Effect of sucrose on oxygen uptake of ascorbic acid in a closed aqueous system. J. Agric. and Food Chemistry. 41:259-262

Johnson, J., R. Braddock and C. Chen (1995). Kinetics of ascorbic acid loss and nonenzymatic browning in orange juice serum. experimental rate constants. J. Food Sci. 60:502-503. 
Kannan, A., D. Kane and T. Labuza (1988). Time and temperature effect on stability of morocan processed orange juice during storage. J. Food Sci. 53:1470.

Kanner, J., J. Fishbein., P. Harel and I. Ben-Gare (1982). Storage stability of orange juice concentrate packaged aseptically. J. Food Sci.47:429-436.

Katherine , Z., R. Michael and V. Joost ( 2003 ). The vitamin C content of orange juice packed in an oxygen scavenger material. Food Chemistry, 82:387-395.

Lee, H. and T. Labuza (1975). Destruction of ascorbic acid as a function of water activity. J. Food Sci.40:370-373.

Liao, M.and P. Seib ( 1988 ).Chemistry of L-ascorbic acid related to foods. Food Chemistry.30:289-312.

Lozano, J. (1991). Kinetics of nonenzymatic browning in model systems simulating clarified apple juice. Lebensm. Wiss. Technol. 24:335-360.

Maria, C., A. Femanda, C. Jorge and M. Jesus (2001). Modeling ascorbic acid thermal degradation and browning in orange juice under aerobic conditions. J. Food Sci and Technology.36(3):303.

Nagy, S., R. Rousef, J. Fisher and H. Lee (1992). "HPLC" Separation and spectral characterization of browning pigments from white grapefruit juice stored in glass and can. J. Agric. Food Chem. 40(1):27.

Navarro, O., R. Perez, F. Gasque and B. Lafuente (1980). Stability of orange Juice concentrate during aseptic refrigerated storage. Revista Deag-Roquimicay Tecnologia de Alimentos.20:389-398.

Nuray, K., S. Handel and K. Feryal (2003). Kinetic of nonenzymatic browning reaction in citrus juice concentrates during storage. Turk. J. Agric.27:353-360.

Petriella,C. ,S .Resnik. and J.Lozano(1985). Kinetic of deteriorative reaction in model food system in high water activity .Color changes due to nonenzymatic browning.J.FoodSci.50:622-626.

Ranganna, S. (1977). Manual of analysis of fruit and vegetabel product.Tata McGraw HiII, New Delhi.

Robertson, G and C. Samaniego (1990). Effect of initial dissolved oxygen levels on the degradation of ascorbic acid and the browning of lemon juice during storage. J. Food Sci.51(1):184.

Roig, M., J. Bello, Z. Rivera, L. Lioyd and J. Kennedy (1999). Studies on the occurrence of nonenzymatic browning during storage of citrus juice. Food Res.Int.32(9):609.

Rojas, A. and L. Gerschenson (1997). Ascorbic acid destruction in sweet aqueous model system. Lebensm. Wiss. Technol. 30(6):567-572.

Roos, Y. and M. Himberg (1994). Nonenzymatic browning behavior as related to glass transition of food model at chilling temperatures. J. Agric and Food Chem.42:893898.

Saguy, I., I. Kopelman and S. Mizrahi (1987). Simulation of ascorbic acid stability during heat processing and concentration of grapefruit juice. J. Food Proc. Eng. 2:213-225.

Sakai, Y., H. Watanabe and R. Takai (1987).A kinetic model for oxidation of ascorbic acid and beta-carotene. J. Food Proc. and Preservation.11:197-207.

Samuel, H. and B. Jerome (1974). Fundamentals Of Physical Chemistry. Macmillan Co. New York. USA.

Singh, R., D. Lund and F. Buelow (1983). Storage stability of intermediate moisture apples: Kinetics of quality change. J. Food Sci.48:939-944.

Tannebaum, S., M. Archer and V. Young ( 1985). Vitamin and minerals. In. O. R. Fennema (Ed). Food Chemistry $\left(2^{\text {ed }}\right)$ (p.488-493). New York: Marcel Dekker.

Wong, M. and D. Stanton (1989). Nonenzymatic browning in kiwifruit juice concentrate system during storage. J. Food Sci.54(3):669. 